\title{
Translingual Neurostimulation (TLNS): A Novel Approach to Neurorehabilitation
}

\author{
Danilov $Y^{*}$ and Paltin D \\ Department of Kinesiology, University of Wisconsin, USA \\ *Corresponding author: Yuri Danilov, Department of \\ Kinesiology, University of Wisconsin, USA
}

Received: May 23, 2017; Accepted: J une 27, 2017;

Published: July 04, 2017

\begin{abstract}
CN-NINM technology represents a synthesis of a new non-invasive brain stimulation technique with applications in physical medicine, cognitive, and affective neurosciences. Our new stimulation method appears promising for the treatment of a full spectrum of movement disorders, and for both attention and memory dysfunction associated with traumatic brain injury. The integrated CN-NINM therapy proposed here aims to restore function beyond traditionally expected limits by employing both newly developed therapeutic mechanisms for progressive physical and cognitive training - while simultaneously applying brain stimulation through a portable neurostimulation device PoNS ${ }^{\text {TM }}$. Based on our previous research and recent pilot data, we believe a rigorous in-clinic CN-NINM training program, followed by regular at-home exercises that will also be performed with CN-NINM, will simultaneously enhance, accelerate, and extend recovery from multiple impairments (e.g. movement, vision, speech, memory, attention, and mood), based on divergent, but deeply interconnected neurophysiological mechanisms of neuroplasticity.
\end{abstract}

Keywords: Neurorehabilitation; Neuromodulation; Translingual Neurostimulation; PoNS Device; Targeted Therapy; Cranial nerve; Neuroplasticity

\section{Abbreviations}

TLNS: Translingual Neurostimulation; CN-NINM: CranialNerve Non-Invasive Neurostimulation; TNS: Trigeminal Nerve Stimulation; VNS: Vagal Nerve Stimulation

\section{Introduction}

The goal of the current paper is to introduce our approach to neurorehabilitation called Cranial Nerve Non-Invasive Neuromodulation (CN-NINM) technology. CN-NINM is a method of intervention that combines Translingual Neurostimulation (TLNS), the Portable neurostimulation Stimulator $\left(\mathrm{PoNS}^{\mathrm{Tn}}\right)$ device, and targeted training designed for movement control rehabilitation.

The basic principles of CN-NINM technology, as a platform technology, build foundation for the development of future directions of neurorehabilitation such as a headache, tinnitus, sleep, depression, etc., using neurostimulation to access brain networks through the cranial nerves, such as those found in the tongue. It is noteworthy, that the principles and corresponding treatment regimens, based on CN-NINM technology, were already successfully implemented for neurorehabilitation of other neurological conditions such as balance, gait, eye movement control, speech and cognitive functions $[1,2]$. Therefore, CN-NINM technology should be considered as a practical realization of several theoretical concepts, based on recent scientific discoveries in the field of neuroscience.

First, we would like to consider abnormal neurological conditions, in the view of modern network science, that result from disruption in similar brain networks. The current understanding of neural-network organization can describe the variety of structural and functional network changes in many neurological and psychiatric diseases, especially in dementia, epilepsy and schizophrenia, but also in traumatic brain injury (TBI), Parkinson's disease, multiple sclerosis (MS), cerebrovascular disease, coma and many other conditions categorized as neuronal network disorders [3-5].

The complexly distributed neuronal network, with multiple cortical and subcortical components, is the physical substrate for any sensory, motor and sensory-motor integrative system, providing, in turn, normal physiological or behavioural function - vision, hearing, postural and eye movement control and multiple others. Damage to or malfunction of any part of said functional network leads to dysfunction of the whole sensory-motor system (spatial and/or temporal abnormalities) that frequently manifests as clinical symptoms.

Second, the situation with the rehabilitation of many neurological symptoms is very similar. Neurological disorders, like TBI, stroke, neurodegenerative disorders or drug overdose (chemical trauma), can affect many distributed networks on many different levels in many different locations. So far, it is almost impossible to identify the exact place and extent of such damages or the extent of malfunctioning tissues, as a result of abnormal connectivity with damaged areas. The abnormalities in the functional relationship between areas and structures, and the abnormalities in the spatio-temporal organization of separate neurons and clusters of neurons are still beyond our reach for assessment and evaluation. As a result of such uncertainty, the therapeutic and rehabilitation resources are significantly limited. For example, there are no effective rehabilitation programs for chronic stage patients after stroke and TBI; the majority of MS symptoms are considered non-recoverable; and there is no effective treatment for 
tinnitus. Physical therapy can help these conditions to some extent, but not dramatically.

TLNS technology was originally designed to modulate complex networks for the purpose of neurorehabilitation. We started from the balance sensory-motor integration network, specifically from postural control rehabilitation after peripheral vestibular damages [68]. Later we extended our approach to the proprioceptive component of balance (multiple sclerosis, amputee), to gate control rehabilitation (Parkinson's disease, MS, TBI, stroke, cerebral palsy), and eye movement control. The combination of neurostimulation (using the PoNS ${ }^{\mathrm{TM}}$ device) and targeted therapy (a set of challenging exercises, explicitly targeting the affected network) became the mainframe of TLNS therapy that is applicable to rehabilitation of many neurological disorders, so far mainly considered untreatable $[1,2,9]$.

\section{Neurostimulation}

Although brain stimulation is well known since ancient Greek and Roman times, from Galen and Scribonius Largus, who used electric eels to treat headaches and various other disorders, the current "explosion" of new neurostimulation methods, devices, and applications are hard to even count. Currently, more than a dozen forms of brain stimulation are undergoing development and evaluation as interventions for neurological and psychiatric disorders [10].

Neurostimulation and neuromodulation techniques are unique forms of treatment distinctly different from pharmacology, psychotherapy, or physical therapy. While these terms are often used interchangeably, for the purpose of this essay and the benefit of this ever-expanding and dynamic field, we propose an important differentiation: Neurostimulation refers to the physical action of stimulating the nervous system, whereas Neuromodulation is the product or result of said stimulation.

\section{Types of neurostimulation}

Specificity and applicability of different neurostimulation methods depend on several key factors: the anatomical location of the stimulation target, physical properties, and the spatio-temporal parameters of stimulation.

The human nervous system is a complex set of interrelated and interacting sub-systems with hierarchical modularity. The modules correspond to major functional systems, such as motor, sensory and association networks. The sub-systems are characterized and called both by their anatomic positions and by their functional specificity.

At the highest level, the nervous system is divided into central and peripheral nervous systems. The central nervous system (CNS) is comprised of the brain and spinal cord and the peripheral nervous system (PNS) incorporates all the remaining neural structures found outside the CNS. The PNS is further divided functionally into the somatic (voluntary) and autonomic (involuntary) nervous systems. The PNS can also be described structurally as being comprised of afferent (sensory) nerves, which carry information toward the CNS, and efferent (motor) nerves, which carry commands away from the CNS [11].

The PNS also consist of spinal nerves and cranial nerves. Although twelve pairs of cranial nerves emerge directly from the brain (anatomically they are part of CNS), and ten pairs of them arise from the brainstem, they are formally considered as a part of PNS.

Correspondingly, all neurostimulation systems can be distinct at the site of application: cranial, spinal cord, spinal ganglion or sciatic nerve neurostimulation systems. It is vital to note that the stimulation of specific brain regions produces equally specific rehabilitation functions

Neurostimulation systems can either be invasive or not invasive. According to the National Institute of Health, non-invasive devices can be defined as those that do not require surgery and do not penetrate the brain parenchyma. Furthermore, the devices for cranial stimulation can be segregated by type of energy source and include, but are not limited to, those used for focused ultrasound stimulation, magnetic seizure therapy, electroconvulsive therapy, static magnets, transcranial alternating current stimulation, transcranial direct current stimulation, transcranial magnetic stimulation, electromagnetic stimulation in radio frequency range, in addition several new systems coming based on optical stimulation of the brain tissue, including infra-red light $[1,12,13]$.

It is important to note that neurostimulation can be external, exogenous, or generated outside of the neural system (transcranial magnetic stimulation, TMS and transcranial direct current stimulation, tDCS) and still attempt to affect excitable neuronal membranes directly by inducing or suppressing neural activity in the brain network [1]. This kind of stimulation is artificial (rather than natural) activation of brain structures by electrical or magnetic fields, or electrical current, or light, or ultrasound (usually applied from outside the body or skull) and is fundamentally different from natural (internal, indigenous) activation.

The natural source of brain activation is neural impulses or spikes that are generated by billions of specialized natural receptors located in depth of skin or internal body tissues. That is internal stimulation from impulses streaming to the spinal cord and brain via nerves and distributed across multiple brain structures [1]. Engagement with natural pathways results in the activation of complex neuronal networks using naturally designed spatial and temporal patterns, unique for different brain structures and based on anatomical and physiological type of neurons, and patterns of interneuron connections. Similar to these processes, are neurostimulation systems that activate the specific receptors, free nerve endings or nerve trunks creating the spike flow. In which case the primary stimulation on the periphery of the neural system is also artificial, but the real factor affecting the CNS is the flow of natural spikes, generated and distributed internally.

\section{Cranial Nerve Neurorehabilitation}

Stimulation

and

One of the major problems of neurorehabilitation is complexity and diversity of the brain's damage. Acquired brain injury (ABI) and neurodegenerative disorders create multiple sites of malfunctioning or physically damaged neural tissue. As a result, various functional systems become inefficient or desynchronized; multiple symptoms developed almost simultaneously. Diversified nature of neural network malfunctions and luck of the methods for localization of such damages become an overwhelming complication for efficient 


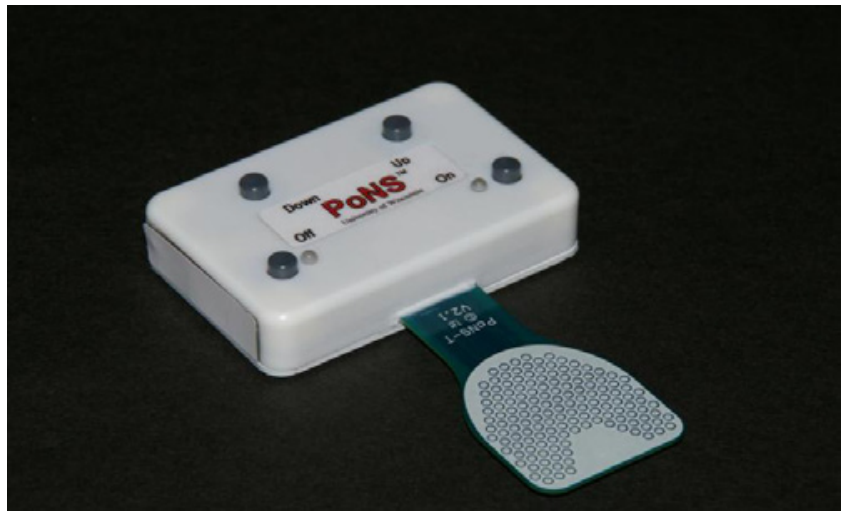

Figure 1: PoNS ${ }^{\mathrm{TM}}$ Version 2.

neurorehabilitation, making full spectrum symptoms and disorders "untreatable."

The majority of existing methods of neurostimulation are limited in several ways. The functional specificity of stimulation creates an extended family of systems for management of selected body parts (bladder) or muscular groups (foot drop). The anatomical specificity and localization of electrodes also restrains efficiency of neurostimulation for functional recovery. The surgical precision of DBS stimulation and the small volume of affected tissue (several cubic millimetres) allow changing activity only in the single node of widely distributed functional network.

The amount of brain tissue affected by TMS, in opposite, might be extended to dozens of cubic centimetres, but activated in an unnatural manner and without functional specificity.

Cranial nerve stimulation might help to solve some these problems. Cranial nerves are the most powerful nerves directly connected to the brain and spinal cord [1]. It is vital to note that all primary sensory systems are streaming information into the CNS. Vision and hearing, smell and taste, vestibular signal and proprioception of the face and tongue continuously directly or indirectly activate the whole brain by cranial nerves.

If we assume, that "multidimensional" damage needs "multidimensional" rehabilitation, then cranial nerve stimulation might be the solution.

TLNS is a unique way to directly and simultaneously activate multiple brain networks by natural spike flow generated on the periphery. The non-invasive and safe "injection" of natural neural activity into damaged neural network initiates the recovery process, based on mechanisms of activity-dependent plasticity.

\section{Existing methods}

The family of cranial nerve stimulation systems is small in comparison with the variety of other neurostimulation systems, and relatively young. The first US FDA approval for vagal nerve stimulation (VNS, Cyberonics, Inc.) was received in 1997. It is a small wonder that the reception of all new methods of neurostimulation, in general, remains controversial and not widely accepted. Many cranial nerve neurostimulation systems are currently under development. The olfactory nerve was not used for neurostimulation purpose yet.
The optic and auditory nerves are mainly under development of various sensory prosthetic devices, for example, artificial retinas and cochlear implants.

However, one system for retina and optic nerve stimulation should be mentioned here: trans-corneal electrical stimulation (TcES) that involves the use of a low-intensity electrical current in the treatment of ophthalmic diseases, including injuries of optic nerve, light-induced photoreceptor degeneration, ocular ischemia, macular dystrophy and retinitis pigmentosa.

Among the others, three pairs of cranial nerves are intensively under investigation for neurorehabilitation purposes: vagal nerve and trigeminal nerve. Both are large, mixed (sensory and motor) cranial nerves.

\section{Vagal Nerve Stimulation (VNS)}

Primary applications for VNS are epilepsy, depression, anxiety, obesity. The target of vagal nerve stimulation (VNS) is the tenth cranial nerve that emerges from the brain at the medulla (brainstem) [13]. It is the longest cranial nerve, extending into the chest and abdominal cavity. Typically, a battery-operated generator is implanted subcutaneously in the left chest wall. An attached electrode is then tunnelled under the skin and wrapped around the left vagal nerve in the neck.

Adverse effects of VNS can be separated into those associated with the complications of the surgery and those resulting from the side effects of stimulation. While risks associated with surgery are minimal, they remain important considerations for both clinicians and patients [13].

There is one non-invasive method, which transcutaneously stimulates the auricular branch of the vagal nerve. It was developed for the treatment of a chronic migraine (NEMOS ${ }^{\circ}$, Cerbomed, Erlangen, Germany). A recent study provides evidence that stimulation using NEMOS at $1 \mathrm{~Hz}$ for four hours daily is effective for chronic migraine prevention over three months $[14,15]$.

\section{Trigeminal Nerve Stimulation (TNS)}

TNS targets the upper, ophthalmic branches of the trigeminal nerve. There are two devices, NeuroSigma and Cephaly, which were originally developed to treat drug resistant epilepsy and sleep disorder, respectively. Side effects of NeuroSigma were mild and included skin irritation, tingling, forehead pressure, and headache [16]. Miller et al. [17] found no side or adverse effects from using Cephaly, which is consistent with our experience using the PoNS ${ }^{\mathrm{TM}}$ device.

\section{PoNS ${ }^{\mathrm{TM}}$ Device}

The PoNS ${ }^{\text {Tx }}$ device, both versions 2 and 4 (Figure $1 \& 2$ respectively), achieves localized electrical stimulation of afferent nerve fibres on the dorsal surface of the tongue via small surface electrodes. Because of the resulting tactile sensation, which, depending on stimulation waveform typically feels like vibration, mild tingling, or pressure; it is certain that tactile nerve fibres are activated. Taste sensations are infrequently reported, although it is not known whether gustatory afferents are in fact stimulated, given the non-physiological patterns of activation likely to result from PoNS-induced stimulation of these fibres [1]. 


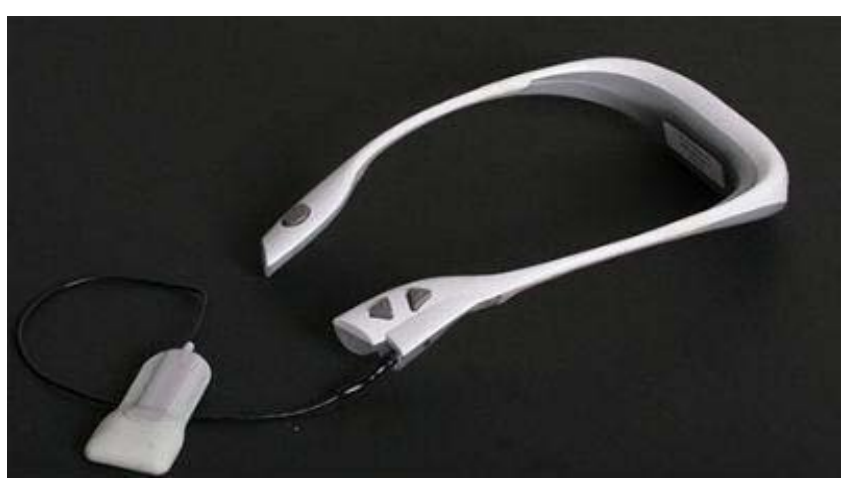

Figure 2: PoNS ${ }^{\mathrm{TM}}$ Version 4.

All electrotactile systems, including the PoNS ${ }^{\mathrm{mi}}$ device, must adhere to a set of core principles to ensure comfortable and controllable tactile precepts, as well as safe operation. As these have already been extensively reviewed $[18,19]$. We will focus on the application of these principles specifically to the PoNS ${ }^{\mathrm{TM}}$ device. An expanded discussion of the waveform, electrode, and safety features appropriate for tongue stimulation has been previously published $[1,20]$.

\section{Why the tongue? Part I}

Electrotactile stimulation supplanted the vibro-tactile stimulation because it is simpler, lighter, consumes less energy, and is easier to control the stimulus. Various improvements have led to the current system. It is an example of a new generation of sensory substitution devices based on computer-controlled electrical stimulation of the human skin in the most densely innervated tactile areas: the tongue and the fingers. The tongue was preferable because it affords a better environment (constant acidity level $(\mathrm{pH})$, constant temperature and humidity, and low excitability thresholds) in comparison with fingertip (variable hydration, thickness of the skin, surface contaminants, relatively limited and highly curved surface area available for stimulation, and high excitability thresholds).

\section{Translingual Neurostimulation (Tlins)}

\section{How the PoNS works}

In brief, TLNS uses sequenced patterns of electrical stimulation on the anterior dorsal surface of the tongue to stimulate the trigeminal and facial nerves. From a technical point of view, the electrical stimulation of the tongue skin by PoNS ${ }^{\mathrm{TM}}$ device is, probably, one of the safest. In each particular moment only set of 9 out of 143 PoNS ${ }^{\mathrm{TM}}$, electrodes are active, surrounded by ground electrodes (16 possible sets in total). Each electrode creates the area of activation $-1.77 \mathrm{~mm}^{2}$. The set of nine electrodes activates total area of $15.9 \mathrm{~mm}^{2}$ or $0.16 \mathrm{~cm}^{2}$. That is a maximum area of the tongue surface activated by PoNS ${ }^{\mathrm{TM}}$ in the single moment.

The signal pattern on each electrode is a sequence of very short rectangular impulses (fixed $19 \mathrm{~V}$ value) with duration of each from 0.4 to 60 microseconds. For comparison, each natural neural impulse (spike duration) is 1 millisecond $=1000$ microseconds $(1.5-3$ milliseconds is a length of full spike waveform).

Considering the pattern of stimulation (sequence of triplet bursts) total stimulation time for single electrodes is in a range from 0.1 to 11 seconds, during typical PoNS ${ }^{\mathrm{TM}}$ application for 20 minutes. Due to the multiplexed nature of the stimulation, each set of 16 electrodes, therefore, delivers a total stimulation time of 1 to 173 seconds during one full session.

The depth of stimuli penetration in the PoNS ${ }^{\mathrm{TM}}$ device is fixed because perceptual intensity is regulated by signal duration, not by current or voltage. The normal thickness of the human tongue epithelia varies from 400 to 800 microns $(0.4-0.8 \mathrm{~mm})$. In the deeper layers, there are muscular fibres, which are moving the human tongue. Electrical stimulation of such fibres creates very distinct sensation of jerking movements of the tongue surface. Our subjects never reach such sensation, considering instructional limit up to "maximal comfortable level." So, we can suggest that the depth of real activation is about $400-600$ micron $(0.4-0.6 \mathrm{~mm})$ range. Then total volume of electrically activated tissue in each particular moment is for one electrode $-0.53-1.06 \mathrm{~mm}^{3}$, for the set of 9 electrodes -4.78 - $9.56 \mathrm{~mm}^{3}$. Therefore, from a technical point of view, the set of 9 electrodes PoNS ${ }^{\mathrm{TM}}$ device activate $0.16 \mathrm{~cm}^{2}$ in area and $5-10 \mathrm{~mm}^{3}$ of tissue volume in each particulate moment, but not more than 154 seconds during 20 minutes' session.

\section{Why the tongue? Part II}

The anterior dorsal surface of the tongue is a patch of the human skin with a unique innervation pattern. The relatively thin (in comparison to other skin areas) oral epithelium is saturated by a different kind of mechanic, there- and taste receptors in addition to free nerve endings, stratified in its depth. It is the area with the maximal density of mechanoreceptors, and, like the fovea in the retina, have the minimum two-point discrimination threshold 0.5-1 mm for mechanical stimulation [21] and $0.25-0.5 \mathrm{~mm}$ for electrotactile stimulation (unpublished data). The physical density, spatial distribution, size of the receptive fields and their overlapping coefficient, spatial and temporal summation properties are largely unknown, especially for electrotactile stimulation [22].

The two major nerves from the tip of the tongue deliver information streams directly to the brainstem - the lingual nerve (the texture of food) and chorda tympani (taste of food). According to our approximation, approximately 20-25 thousand neural fibers deliver neural impulses from this area (about $7.5 \mathrm{~cm}^{2}$ ) covered by PoNS ${ }^{\mathrm{TM}}$ electrode array.

\section{CN-NINM technology platform}

It is important to clarify that CN-NINM is a platform that consist of many technologies, all of which target cranial nerves-primarily Vagal and Trigeminal-with the intention of influencing the central nervous system. NeuroSigma, Cefaly, NEMOS, and SIMPATOCOR are examples of other technologies within the CN-NINM canon. TLNS, using the PoNS ${ }^{\mathrm{TM}}$ device, is a novel class of stimulation to join this existing platform.

However, these other stimulation devices and techniques target their stimulation to nerve trunks. TLNS alternatively targets the receptors and nerve endings. In his way, TLNS is closer to natural stimulation than the other techniques because synchronous stimulation of nerve receptors is a more natural input than stimulation of nerve trunks. 


\section{Induced Neuroplasticity}

Our hypothesis is that TLNS induces neuroplasticity by noninvasive stimulation of two major cranial nerves: trigeminal, $\mathrm{CN}-\mathrm{V}$, and facial, CN-VII. This stimulation excites a natural flow of neural impulses to the brainstem (pons varolli and medulla), and cerebellum via the lingual branch of the cranial nerve $(\mathrm{CN}-\mathrm{Vc})$, and chorda tympani branch of CN-VII, to effect changes in the function of these targeted brain structures [23].

The spatio-temporal trains of neural activation induced in these nerves eventually produce changes of neural activity in corresponding nuclei of the brainstem - at least in the sensory and spinal nuclei of trigeminal nuclei complex (the largest nuclei in the brainstem, extending from the midbrain to the nuclei of the descending spinal tracts), and the caudal part of the nucleus tractus solitarius, cochlear, cuneate and hypoglossal nuclei and upper segment of the spine (C1C3), where both stimulated nerves to have direct projections.

Changes in neural activity were evident in the results of our pilot study, wherein we also developed a new fMRI signal processing method to yield high-resolution images of the pons, brainstem and cerebellum beyond that previously reported, allowing observation of changes in functional activity in all of the regions of interest [24-26]. We are particularly interested in these specific changes (in the pons, brainstem, and cerebellum) because these neural structures are the major sensory integration and movement control centres of the brain and therefore primary targets for neuromodulation.

We postulate that the intensive activation of these structures initiates a sequential cascade of changes in neighbouring and/or connected nuclei by direct projections and collateral connections, by activation of brainstem interneuron circuitries (reticular formation of the brainstem), and/or by passive transmission of biochemical compounds in the intercellular space (release of neurotransmitters in the synaptic gaps). The stream of neural impulses leads to activation of corresponding neural networks and massive release of neurotransmitters that eventually activate the glial networks of the brainstem (responsible for maintenance of neuronal environment and synaptic gaps).

This, in turn, causes radiating therapeutic neurochemical and neurophysiological changes affecting both synaptic and extrasynaptic circuitries affecting information processing of afferent and efferent neural signals involved in movement control, including the cerebellum and nuclei of spinal motor pathways.

The temporal pattern of our observed retention effects is strikingly similar to the process well known in neuroscience literature for several decades as long-term potentiation (LTP) and depression (LTD). Both processes were tested and verified in multiple animal models by analysing changes in brain tissue samples, and both are in intensive use in different models of human processes of learning and memory as a basic mechanism of the synaptic plasticity of the brain [27-31].

In brief, synaptic plasticity is a natural manifestation of activity-dependent processes affecting structure and function of multiple neuronal networks. As a result of such processes, multiple consequential adaptive changes are happening on different levels of brain organization (molecular, cellular, regional, and systemic), with different temporal patterns and dynamics (short and long) that reflects on multiple sensory and motor functions, cognitive performance and behaviour [32-34].

Intensive repetitive stimulation of neurons leads to the corresponding activation of synaptic contacts on the axonal tree, including the whole complex of pre- and post- synaptic neurochemical mechanisms. Multiphasic fluctuations of postsynaptic potentials, frequently described as short-term activity-dependent synaptic plasticity (in range milliseconds, seconds and minutes) has been shown capable of enhancing synaptic transmissions $[35,36]$.

In contrast, long-term potentiation (LTP) is the phenomena of synaptic structural remodelling and formation of new synaptic contacts that is activated by high frequency stimulation [37-42]. After $10-40$ minutes of high-frequency stimulation $(50-400 \mathrm{~Hz}$, range of frequencies used in animal research) the number of synapses and proportion of multiple spine boutons can increase the efficiency of neural connections. Effects of LTP can continue during several hours and even days [43].

In our experiments, using the PoNS device, prolonged and repetitive activation (20 minutes or more) of functional neuronal circuits (balance, gait) can initiate long-lasting processes of neuronal reorganization, (similar to LTP), that we can see and measure in subjects' behaviour. The functional improvement after initial training sessions continues for several hours. Multiple regular sequential training sessions lead to the consistent increase of improved symptom duration and cumulative enhancement of affected functions.

This regular excitation may also increase the receptivity of numerous other neural circuitries and affect internal mechanisms of homeostatic self-regulation, according to the contemporary concept of synaptic plasticity. We cannot also exclude that this induces simultaneous activation of serotonergic and noradrenergic regulation systems of the brain as well.

The result of this intervention is essentially brain plasticity on demand - priming or up-regulating of targeted neural structures to develop new functional pathways, which is the goal of neurorehabilitation and a primary means of functional recovery from permanent physical damage caused by stroke or trauma.

The effectiveness of TLNS was demonstrated in multiple case studies (more than 300 subjects) during the last ten years. In brief, statistically significant improvement in balance and gait was recorded in: the MS pilot study (13 subjects); the balance and gait in MS control study (10/10 subjects); the pilot study of balance disorders (23 subjects), the balance in pilot stroke study (5 subjects), traumatic brain injury ( 45 subjects).

The independent control study of the effect of TLNS on balance and gait in MS subjects (7/7) was conducted in Montreal Neurological Institute and Hospital (MNIH). The comparison of fMRI images before and after TNLS revealed significant changes in the activity of cortical areas responsible for gait in the active group vs. control group. Surprisingly, significant changes in BOLD signals were also present in areas responsible for working memory (dorsolateral prefrontal cortex, DLPFC and right anterior cingulate cortex, rACC). Results are in press. 
Significant improvement in balance and gait using TLNS stimulation was found and reported in a subject with chronic spinal cord injury children with posterior fossa, cerebral palsy (45/20).

\section{Summary}

In a sense, TLNS is an effective combination of several existing neurostimulation techniques. It stimulates the trigeminal nerve, similar to eTNS and Cefaly, but targets a different branch (V3, instead of V1, the largest branch of the trigeminal nerve). Also, it simultaneously stimulates the facial nerve (chorda tympani) branch and correspondingly solitary nuclei, as VNS does, but non-invasively. Furthermore, we are observing activation of the ventral cerebellum, as result of tongue stimulation. There is solid evidence from animal research that stimulation of anterior third of the tongue can activate directly and antidromically the hypoglossal nuclei. There are human anatomical data, supporting the hypothesis, which TNLS might be considered as a soft, non-invasive version of DBS.

Granted, this cranial nerve neurostimulation technology is coming through its first painful steps of development. Much more studies, controlled and blinded should be done, new problems and solutions should be discovered, before we will have a better understanding of the mechanisms of action for TLNS.

\section{Conclusions}

The cranial nerves neurostimulation is a new, small, but distinct set of technologies among the wide family of peripheral nerve stimulation methods that represent a unique approach to neurorehabilitation of multiple disorders and the wide spectrum of malfunctions in the human central nervous system.

As an example of safe, non-invasive, easy to manage, patientoriented technology, TLNS can be considered as an alternative way to approach previously untreatable symptoms and conditions, like the chronic stage of TBI and stroke.

The PoNS ${ }^{\mathrm{TM}}$ device is the ideal clinical tool. The scope of clinical applications will continue to grow because of several unique characteristics: it is multi-directional, effective, non-invasive, safe, and the stimulation is repeatable and easy to control. None of the described harms, typical of other invasive and non-invasive neurostimulation methods are not applicable to TLNS. Moreover, the majority of the side effects produced by existing clinical devicesare targets for rehabilitation and improvement for our technology. We have never observed an effect of overstimulation or "overdose" with the PoNS ${ }^{\mathrm{TM}}$ or any negative effects. However, minor, episodic discomfort or mild headache episodes during developmental or adaptive stages should be noted.

The physiological nature and network based principals of TNLS make it a good match to currently developing a point of view on neural network origin of many neurological disorders and recovery of functional systems, as an appropriate way of neurorehabilitation.

\section{Disclosure}

The author has a financial interest in Advanced Neuro Rehabilitation LLC and in Helius Medical Technologies., which both have intellectual property rights in the field of use reported in this article.

\section{References}

1. Danilov Y, Kaczmarek K, Skinner K, Tyler M. Cranial Nerve Noninvasive Neuromodulation: New Approach to Neurorehabilitation. In Brain Neurotrauma: Molecular, Neuropsychological, and Rehabilitation Aspects. 2015; 605-628. CRC Press.

2. Tyler ME, Kaczmarek KA, Rust KL, Subbotin AM, Skinner KL, Danilov YP. Non-invasive neuromodulation to improve gait in chronic multiple sclerosis: a randomized double blind controlled pilot trial. Journal of neuroengineering and rehabilitation. 2014; 11: 79

3. Filippi M, van den Heuvel MP, Fornito A, He Y, Pol HE, Agosta F, et al. Assessment of system dysfunction in the brain through MRI-based connectomics. The Lancet Neurology. 2013; 12: 1189-1199.

4. Pol HH, Bullmore E. Neural networks in psychiatry. European Neuropsycho pharmacology. 2013; 23: 1-6.

5. Stam CJ. Modern network science of neurological disorders. Nature Reviews Neuroscience. 2014; 15: 683-695.

6. Ghulyan-Bedikian V, Paolino M, Paolino F. Short-term retention effect of rehabilitation using head position-based electrotactile feedback to the tongue: Influence of vestibular loss and old-age. Gait \& posture. 2013; 38: 777-783.

7. Robinson BS, Cook JL, Richburg CM, Price SE. Use of an electrotactile vestibular substitution system to facilitate balance and gait of an individual with gentamicin-induced bilateral vestibular hypofunction and bilateral transtibial amputation. Journal of Neurologic Physical Therapy. 2009; 33: 150-159.

8. Tyler M, Danilov Y, Bach-y-Rita P. Closing an open-loop control system: vestibular substitution through the tongue. Journal of integrative neuroscience. 2003; 2: 159-164.

9. Harbourne R, Becker K, Arpin DJ, Wilson TW, Kurz MJ. Improving the Motor Skill of Children with Posterior Fossa Syndrome: A Case Series. Pediatric Physical Therapy. 2014; 26: 462-468.

10. Danilov YP, Kublanov VS. Emerging Noninvasive Neurostimulation Technologies: CN-NINM and SYMPATOCORECTION. Journal of Behavioral and Brain Science. 2014; 2014

11. Harry JD, Niemi JB, Kellogg S, D'Andrea S, inventors; Trustees Of Boston University, assignee. System and method for neuro-stimulation. United States patent US 9,616,234. 2017

12. Neren D, Johnson MD, Legon W, Bachour SP, Ling G, Divani AA. Vagus nerve stimulation and other neuromodulation methods for treatment of traumatic brain injury. Neurocritical care. 2016; 24: 308-319.

13. George MS, Aston-Jones G. Noninvasive techniques for probing neurocircuitry and treating illness: vagus nerve stimulation (VNS), transcranial magnetic stimulation (TMS) and transcranial direct current stimulation (tDCS). Neuropsychopharmacology. 2010; 35: 301-316.

14. Straube A, Ellrich J, Eren O, Blum B, Ruscheweyh R. Treatment of chronic migraine with transcutaneous stimulation of the auricular branch of the vagal nerve (auricular t-VNS): a randomized, monocentric clinical trial. The journal of headache and pain. 2015; 16: 63.

15. Straube A, Eren O, Gaul C. Role of the vagal nerve in the pathophysiology and therapy of headache. MMW Fortschritte der Medizin. 2016; 158: 74-76.

16. Soss J, Heck C, Murray D, Markovic D, Oviedo S, Corrale-Leyva G, et al. A prospective long-term study of external trigeminal nerve stimulation for drugresistant epilepsy. Epilepsy \& Behavior. 2015; 42: 44-47.

17. Miller S, Sinclair AJ, Davies B, Matharu M. Neurostimulation in the treatment of primary headaches. Practical neurology. 2016; 16: 362-375.

18. Kaczmarek, KA, Bach-Y-Rita P. Tactile displays. Virtual environments and advanced interface design. 1995; 55: 349.

19. Szeto AY, Saunders FA. Electrocutaneous stimulation for sensory communication in rehabilitation engineering. IEEE Transactions on Biomedical Engineering. 1982: 300-308.

20. Kaczmarek KA. The tongue display unit (TDU) for electrotactile spatiotemporal 
pattern presentation. Scientia Iranica. 2011; 18: 1476-1485

21. Vallbo ÅB, Johansson RS. Properties of cutaneous mechanoreceptors in the human hand related to touch sensation. Hum Neurobiol. 1984; 3: 3-14.

22. Johansson RS, Vallbo ÅB. Tactile sensibility in the human hand: relative and absolute densities of four types of mechanoreceptive units in glabrous skin. The Journal of physiology. 1979; 286: 283-300.

23. Wildenberg JC, Tyler ME, Danilov YP, Kaczmarek KA, Meyerand ME. Sustained cortical and subcortical neuromodulation induced by electrical tongue stimulation. Brain imaging and behavior. 2010; 4: 199-211.

24. Wildenberg JC, Tyler ME, Danilov YP, Kaczmarek KA, Meyerand ME. Electrical tongue stimulation normalizes activity within the motion-sensitive brain network in balance-impaired subjects as revealed by group independen component analysis. Brain connectivity. 2011; 1: 255-265

25. Wildenberg JC, Tyler ME, Danilov YP, Kaczmarek KA, Meyerand ME. Highresolution $\mathrm{fMRI}$ detects neuromodulation of individual brainstem nuclei by electrical tongue stimulation in balance-impaired individuals. Neuroimage. 2011: 56: 2129-2137.

26. Wildenberg JC, Tyler ME, Danilov YP, Kaczmarek KA, Meyerand ME. Altered connectivity of the balance processing network after tongue stimulation in balance-impaired individuals. Brain connectivity. 2013; 3: 87-97.

27. Bear MF, Malenka RC. Synaptic plasticity: LTP and LTD. Current opinion in neurobiology. 1994; 4: 389-399.

28. Dudek SM, Bear MF. Bidirectional long-term modification of synaptic effectiveness in the adult and immature hippocampus. Journal of Neuroscience. 1993; 13: 2910-2918.

29. Kirkwood A, Lee HK, Bear MF. Co-regulation of long-term potentiation and experience-dependent synaptic plasticity in visual cortex by age and experience. Nature. 1995; 375: 328.

30. Larkman AU, Jack JJ. Synaptic plasticity: hippocampal LTP. Current opinion in neurobiology. 1995; 5: 324-334.

31. Levenes C, Daniel H, Crépel F. Long-term depression of synaptic transmission in the cerebellum: cellular and molecular mechanisms revisited. Progress in neurobiology. 1998; 55: 79-91.
32. Shin RM, Tsvetkov E, Bolshakov VY. Spatiotemporal asymmetry of associative synaptic plasticity in fear conditioning pathways. Neuron. 2006; 52: 883-896.

33. Shin RM, Tully K, Li Y, Cho JH, Higuchi M, Suhara T, et al. Hierarchical order of coexisting pre-and postsynaptic forms of long-term potentiation a synapses in amygdala. Proceedings of the National Academy of Sciences. 2010; 107: 19073-19078.

34. Tully K, Li Y, Bolshakov VY. Keeping in check painful synapses in centra amygdala. Neuron. 2007; 56: 757-759.

35. Zucker RS. Calcium-and activity-dependent synaptic plasticity. Current opinion in neurobiology. 1999; 9: 305-313.

36. Zucker RS, Regehr WG. Short-term synaptic plasticity. Annual review of physiology. 2002; 64: 355-405.

37. Buchs PA, Muller D. Induction of long-term potentiation is associated with major ultrastructural changes of activated synapses. Proceedings of the National Academy of Sciences. 1996; 93: 8040-8045.

38. Calverley RK, Jones DG. Contributions of dendritic spines and perforated synapses to synaptic plasticity. Brain research reviews. 1990; 15: 215-249.

39. Engert F, Bonhoeffer T. Dendritic spine changes associated with hippocampal long-term synaptic plasticity. Nature. 1999; 399: 66-70.

40. Geinisman Y, Berry RW, Disterhoft JF, Power JM, Van der Zee EA. Associative learning elicits the formation of multiple-synapse boutons. Journal of Neuroscience. 2001; 21: 5568-5573.

41. Jones DG, Itarat W, Calverley RK. Perforated synapses and plasticity Molecular neurobiology. 1991; 5: 217-228

42. Toni N, Buchs PA, Nikonenko I, Bron CR, Muller D. LTP promotes formation of multiple spine synapses between a single axon terminal and a dendrite. Nature. 1999; 402: 421-425

43. Bliss TV, Lømo T. Long-lasting potentiation of synaptic transmission in the dentate area of the anaesthetized rabbit following stimulation of the perforant path. The Journal of physiology. 1973; 232: 331-356
Phys Med Rehabil Int - Volume 4 Issue 2 - 2017

ISSN : 2471-0377 | www.austinpublishing group.com

Danilov et al. (C) All rights are reserved
Citation: Danilov Y and Paltin D. Translingual Neurostimulation (TLNS): A Novel Approach to Neurorehabilitation. Phys Med Rehabil Int. 2017; 4(2): 1117. 